\title{
COMPUTABILITY OF FØLNER SETS
}

\author{
MATTEO CAVALERI
}

\begin{abstract}
We define the notion of computability of Følner sets for finitely generated amenable groups. We prove, by an explicit description, that the Kharlampovich groups, finitely presented solvable groups with unsolvable Word Problem, have computable Følner sets. We also prove computability of Følner sets for extensions -with subrecursive distortion functions- of amenable groups with solvable Word Problem by finitely generated groups with computable Følner sets . Moreover we obtain some known and some new upper bounds for the Følner function for these particular extensions.
\end{abstract}

\section{INTRODUCTION}

In this paper we define and study an effective version of amenability for finitely generated groups in terms of computability of Følner sets. Let $\Gamma$ be a group generated by a finite set $X$. We denote by $\pi_{\Gamma, X}: \mathbb{F}_{X} \rightarrow \Gamma$ the canonical epimorphism from the free group over $X$ to $\Gamma$. For any $n \in \mathbb{N}$, an $n$-Følner set for $\Gamma$ (with respect to $X$ ) is defined to be a non-empty finite subset $F \subset \Gamma$ such that

$$
\frac{|F \backslash x F|}{|F|} \leq n^{-1}, \quad \forall x \in X
$$

$\Gamma$ is amenable if it admits $n$-Følner sets, for all $n \in \mathbb{N}$. In order to make effective this notion, we simply ask for computability of finite preimages of Følner sets in the covering free group.

Definition A. $\Gamma$ has computable Følner sets with respect to $X$ if there exists an algorithm with:

INPUT: $n \in \mathbb{N}$

OUTPUT: $F \subset \mathbb{F}_{X}$ finite, such that $\pi_{\Gamma, X}(F)$ is $n$-Følner for $\Gamma$.

This definition does not depend on the particular choice of the finite set of generators (see Proposition 1$)$.

The Følner function $F_{\Gamma, X}$ of $\Gamma$ (with respect to $X$ ) was defined by Vershik [19] by

$$
F_{\Gamma, X}(n):=\min \{|F|: \quad F \subset \Gamma \text { is } n \text { - Følner }\} .
$$

2010 Mathematics Subject Classification. 20F10, 03D40, 43 A07.

This work was partially supported by a grant of the Romanian National Authority for Scientific Research and Innovation, CNCS - UEFISCDI, project number PN-II-RU-TE-2014-4-0669, and partially supported by the European Research Council (ERC) grant ANALYTIC no. 259527 of Prof. Goulnara Arzhantseva. 
The aim of our investigation, and in particular of Definition A, is to formalize and answer an old question, in our notation:

Question (Vershik). "is it possible, in some sense, to (algorithmically) describe the $n$-Folner sets of $\Gamma$ even if there is no solution for the Word Problem?"

This question arose after the construction by Kharlampovich of finitely presented groups, solvable and therefore amenable, with unsolvable Word Problem [11] (following the notation in [13], let denote them by $G(M)$ ).

Indeed a finitely generated amenable group with solvable Word Problem has computable Følner sets: for every $n \in \mathbb{N}$ we can enumerate all finite subsets of $\mathbb{F}_{X}$ and for each subset check, by solvability of the Word Problem, condition (1), until we find the preimage of an $n$-Følner set: the algorithm will eventually stop because $\Gamma$ is amenable (for details [2,3]).

A first negative answer to Vershik's question was given by Erschler [7], providing examples of finitely generated groups with Følner functions growing faster than any given function (a result recovered in $[10,17])$ : thus, when the given function is not subrecursive (i.e. without any recursive upper bound) there is no hope to algorithmically describe Følner sets of the associated groups (for details [2,3]).

On the other hand, in [3], we proved that, if $\Gamma$ is recursively presentable and amenable then

(i) the Følner function of $\Gamma$ is subrecursive;

(ii) there exists an algorithm computing the Reiter functions of $\Gamma$;

(iii) computability of one-to-one preimages of Følner sets is equivalent to solvability of the Word Problem.

As a consequence, for recursively presented amenable groups, the notion of computability of Følner sets is the only non trivial notion of effective amenability that is not characterized by solvability of the Word Problem.

Indeed computability of Følner sets does not imply solvability of the Word Problem: this is the first consequence of the following theorem, proved by a very explicit description of the Følner sets of $G(M)$ (see Section 1).

\section{Theorem A. The Kharlampovich groups $G(M)$ have computable Følner sets.}

Amenability is stable under semidirect products and, more generally, under extensions: in the literature, the most common proofs of these two facts do not use the characterization of amenability by Følner sets. The book [5] is one of the exceptions and it was a valuable resource for our proofs. Moreover in [12] it was explicitly shown that a Følner net for the semidirect product 
is given by the product of the Følner nets of the factor groups. However this does not yield an effective procedure to produce, for a fixed $n \in \mathbb{N}$, an $n$-Følner set.

After the Preliminaries, each section consists of a Theorem about the general shape of $n$-Følner sets of group extensions, a Corollary about computability of these Følner sets and a Corollary about the Følner functions. We can interpret the results of this paper as stability properties of the class of groups with computable Følner sets and of the class of groups with subrecursive Følner functions.

Section 1. We consider the case of a splitting extension by an Abelian group which is finitely generated as a normal subgroup (this is the case of $G(M)$ ): computability of Følner sets and subrecursivity of Følner function are preserved.

Section 2. We consider general Abelian extensions: subrecursivity of Følner functions is preserved but we prove computability of Følner sets just if the quotient group has solvable Word Problem. We don't know if this hypothesis is necessary. Asymptotically equivalent bounds for the Følner function of solvable groups could be also deduced from $[7,8]$, or using the comparison with the Følner function in free solvable groups in [18].

Section 3. We consider the semidirect product between two finitely generated groups: if both have computable Følner sets then the product has computable Følner sets.

Section 4. We consider an extension $\Gamma$ of a finitely generated group $K$ by a finitely generated group $N=\langle Y\rangle$. If $K$ and $N$ have subrecursive Følner functions and the distortion function $\Delta_{N}^{\Gamma}(n):=\max \left\{|\omega|_{Y}: \omega \in N,|\omega|_{X} \leq n\right\}$ is subrecursive, then $\Gamma$ has subrecursive Følner function; if $N$ has computable Følner sets, $K$ is amenable with solvable word problem and $\Delta_{N}^{\Gamma}$ is subrecursive then $\Gamma$ has computable Følner sets. Notice that it is possible that $\Delta_{N}^{\Gamma}$ is not subrecursive, see for example [1], even for solvable groups, see [6]. Again we don't know if these hypotheses are necessary.

\section{Questions.}

1. Have all finitely generated solvable groups computable Følner sets?

2. Is computability of Følner sets stable under quotients?

3. Does subrecursivity of the Følner function imply computability of Følner sets?

A positive answer to the third question would imply both computability of Følner sets for every recursively presented amenable group and a positive answer to the second question, because subrecursivity of the Følner function is stable under quotients (see [7, Lemma 2.2]); a positive answer 
to the second question would imply a positive answer to the first one, because free solvable groups have solvable Word Problem and therefore have computable Følner sets.

Acknowledgements. This work started from my $\mathrm{PhD}$ program in Sapienza Università di Roma, under the supervision of Tullio Ceccherini-Silberstein. Part of this work was developed during the program Measured group theory in Vienna in 2016. We thank its organizers, as well as the Erwin Schrödinger Institute for Mathematics and Physics, for the warm hospitality. We also thank gratefully Federico Berlai for an improvement in Theorem 2.1 and Theorem 4.1, Michel Coornaert for precious comments, Goulnara Arzhantseva and Anatoly Vershik for their interest and encouragement.

\section{PRELIMINARIES}

Throughout this paper $\mathfrak{F} \varnothing l_{\Gamma, X}(n)$ is the family of $n$-Følner sets of $\Gamma$ with respect to $X$. For an element $g \in \Gamma$ we denote with $|g|$ the length with respect to $X \cup X^{-1}$ (so it is the minimal length of a word in $\mathbb{F}_{X}$ representing $g$ ). For a different set of generators, say $Y$, we explicitly write $|g|_{Y}$. For a subset $A$ we also denote with $|A|_{Y}$ the maximal length of the elements of $A$ with respect to $Y$. We denote by $B_{n}$ the ball of radius $n$ in the free group and $B_{n}(\Gamma):=\pi_{\Gamma, X}\left(B_{n}\right)$ the ball of radius $n$ of $\Gamma$.

Lemma 1. For any $F \in \mathfrak{F} \varnothing l_{\Gamma, X}(n)$ and for all $g \in \Gamma$ we have:

$$
\frac{|F \backslash g F|}{|F|} \leq|g| n^{-1}
$$

Proof. At first we observe that if $F \in \mathfrak{F} \varnothing l_{\Gamma, X}(n)$, for every $x \in X$ we have:

$$
\frac{\left|F \backslash x^{-1} F\right|}{|F|}=\frac{\mid x^{-1}(x F \backslash F \mid)}{|F|}=\frac{|x F \backslash F|}{|F|}=\frac{|F \backslash x F|}{|F|} \leq n^{-1} .
$$

If $g=x_{1} \ldots x_{|g|}$, with $x_{1}, \ldots, x_{|g|} \in X \cup X^{-1}$,

$$
\left(F \backslash x_{1} \ldots x_{|g|} F\right) \subset\left[\left(F \backslash x_{1} F\right) \cup\left(x_{1} F \backslash x_{1} x_{2} F\right) \cup \ldots \cup\left(x_{1} \ldots x_{|g|-1} F \backslash x_{1} \ldots x_{|g|} F\right)\right]
$$

and $\left|x_{1} \ldots x_{j-1} F \backslash x_{1} \ldots x_{j} F\right|=\left|F \backslash x_{j} F\right|$.

Proposition 1. Suppose $\Gamma$ has computable Følner sets with respect to $X$. Let $Y \subset \Gamma$ be another finite generating subset. Then $\Gamma$ has computable Folner sets with respect to $Y$ as well.

Proof. By expressing every $x \in X$ in terms of words in $Y$, we define a homomorphism $\phi: \mathbb{F}_{X} \rightarrow \mathbb{F}_{Y}$ such that the following diagram commutes: 


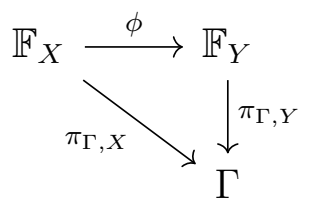

We define the natural number $m:=|\phi(X)|_{Y}$ as the maximum of the word length in $\mathbb{F}_{Y}$ of the image of generators of $\mathbb{F}_{X}$. Suppose $W \subset \mathbb{F}_{X}$ is a finite subset such that $\pi_{\Gamma, X}(W) \in \mathfrak{F} \varnothing l_{\Gamma, X}(m n)$; by Lemma $1 \pi_{\Gamma, Y}(\phi(W))=\pi_{\Gamma, X}(W) \in \mathfrak{F} \varnothing l_{\Gamma, Y}(n)$. Combining the algorithm of Definition A for $X$, with the algorithm computing the homomorphism $\phi$, we deduce the existence of the desired algorithm for $Y$.

Remark 1. In finitely presented case (by Tietze transformations), or, more generally, whenever we can write the old generators in terms of the new ones, we can explicitely update the algorithm of Definition A for the new generators.

Lemma 2. If $\Gamma$ is amenable then there exists $F \in \mathfrak{F} \varnothing l_{\Gamma, X}(n)$ such that $|F| \leq F_{\Gamma, X}(|X| n)$ and $F \subset B_{|F|}(\Gamma)$.

Proof. We define

$$
\mathfrak{F} \varnothing l_{\Gamma, X}^{\prime}(n):=\left\{F, \text { non-empty finite subset of } \Gamma: \frac{\left|\partial_{X} F\right|}{|F|} \leq \frac{1}{n}\right\},
$$

where $\partial_{X} F:=\{f \in F: \exists x \in X: x f \notin F\}$.

It is known and easy to see that if $F^{\prime}$ is of minimal cardinality in $\mathfrak{F} \varnothing l_{\Gamma, X}^{\prime}(n)$ (optimal Følner set) then it is connected as subgraph of the right Cayley graph of $\Gamma$ with respect to $X$ (see [2] for details). In particular for $f \in F^{\prime}$ we have that $1_{\Gamma} \in F:=F^{\prime} f^{-1}$ and $F \subset B_{|F|}(\Gamma)$ and $F \in \mathfrak{F} \varnothing l_{\Gamma, X}^{\prime}(n)$. Finally since:

$$
\mathfrak{F} \varnothing l_{\Gamma, X}(|X| n) \subset \mathfrak{F} \varnothing l_{\Gamma, X}^{\prime}(n) \subset \mathfrak{F} \varnothing l_{\Gamma, X}(n)
$$

we have that $F \in \mathfrak{F} \varnothing l_{\Gamma, X}(n)$ and $|F| \leq F_{\Gamma, X}(|X| n)$.

Definition 1. Let $y_{1}, y_{2}, \ldots, y_{s}$ be pairwise commuting elements of $\Gamma$, not necessarily distinct. Set:

$$
C_{n}\left(y_{1}, y_{2}, \ldots, y_{s}\right):=\left\{y_{1}^{i_{1}} y_{2}^{i_{2}} \ldots y_{s}^{i_{s}}: \quad i_{1}, i_{2}, \ldots, i_{s} \in\{0,1, \ldots, n-1\}\right\}
$$

\section{Lemma 3.}

$$
\frac{\left|C_{n}\left(y_{1}, y_{2}, \ldots, y_{s}\right) \backslash y_{j} C_{n}\left(y_{1}, y_{2}, \ldots, y_{s}\right)\right|}{\left|C_{n}\left(y_{1}, y_{2}, \ldots, y_{s}\right)\right|} \leq n^{-1}, \quad \forall j \in\{1,2, \ldots, d\}
$$


Proof. Since all elements $y_{1}, y_{2}, \ldots, y_{s}$ commute we prove, without loss of generality, the statement for $j=1$.

At first, we observe that $C_{n}\left(y_{1}, y_{2}, \ldots, y_{s}\right)=C_{n}\left(y_{1}\right) C_{n}\left(y_{2}, y_{3}, \ldots, y_{s}\right)$ and

$$
C_{n}\left(y_{1}\right) \backslash y_{1} C_{n}\left(y_{1}\right)=\left\{\begin{array}{l}
\emptyset \text { if } y_{1} \text { has order less than or equal to } n \\
\left\{1_{\Gamma}\right\} \text { otherwise }
\end{array}\right.
$$

Writing $C_{n}$ instead of $C_{n}\left(y_{1}, y_{2}, \ldots, y_{s}\right)$ we have that

$$
C_{n} \backslash y_{1} C_{n} \subset C_{n}\left(y_{2}, y_{3}, \ldots, y_{s}\right)
$$

because $C_{n} \backslash y_{1} C_{n} \subset\left[C_{n}\left(y_{1}\right) \backslash y_{1} C_{n}\left(y_{1}\right)\right] C_{n}\left(y_{2}, y_{3}, \ldots, y_{s}\right)$.

Now we show that $C_{n}$ contains $n$ disjoint translations of $C_{n} \backslash y_{1} C_{n}$, precisely:

$$
C_{n} \supset \bigsqcup_{k=0}^{n-1} y^{k}\left[C_{n} \backslash y_{1} C_{n}\right]
$$

At first

$$
y_{1}^{k}\left[C_{n} \backslash y_{1} C_{n}\right] \subset y_{1}^{k} C_{n}\left(y_{2}, y_{3}, \ldots, y_{s}\right) \subset C_{n}, \quad \forall k \in\{0,1, \ldots, n-1\}
$$

in particular if $g \in y_{1}^{k}\left[C_{n} \backslash y_{1} C_{n}\right]$ there exist $\widehat{i}_{2}, \ldots, \widehat{i_{s}} \in\{0,1, \ldots, n-1\}$ such that $g=y_{1}^{k} y_{2}^{\widehat{i_{2}}} \ldots y_{s}^{\hat{i}_{s}}$. If $k \neq 0$ then $g \notin C_{n} \backslash y_{1} C_{n}$, this implies:

$$
y_{1}^{k}\left[C_{n} \backslash y_{1} C_{n}\right] \cap\left[C_{n} \backslash y_{1} C_{n}\right]=\emptyset, \quad \forall k \in\{1, \ldots, n-1\}
$$

Thus $\left\{y_{1}^{k}\left[C_{n} \backslash y_{1} C_{n}\right]\right\}_{k=0, \ldots, n-1}$ are disjoint sets and (2) is proved and therefore we deduce $\frac{\left|C_{n} \backslash y_{1} C_{n}\right|}{\left|C_{n}\right|} \leq n^{-1}$.

For a finite subset $Y \subset \Gamma$ we may have different finite enumerations of $Y$, for example we consider $W, W^{\prime} \subset \mathbb{F}_{X}, W=\left\{w_{1}, \ldots, w_{t}\right\}$ and $W^{\prime}=\left\{w_{1}^{\prime}, \ldots, w_{t^{\prime}}^{\prime}\right\}$ such that $\pi_{\Gamma, X}(W)=$ $\pi_{\Gamma, X}\left(W^{\prime}\right)=Y$. In general, $C_{n}\left(\pi_{\Gamma, X}\left(w_{1}\right), \ldots, \pi_{\Gamma, X}\left(w_{t}\right)\right) \neq C_{n}\left(\pi_{\Gamma, X}\left(w_{1}^{\prime}\right), \ldots, \pi_{\Gamma, X}\left(w_{t^{\prime}}^{\prime}\right)\right)$ in $\Gamma$ but these subsets are both $n^{-1}$-invariant by left multiplication by every element $y \in Y$, by virtue of Lemma 3. By abuse of notation we simply write $C_{n}(Y)$ instead of $C_{n}\left(\pi\left(w_{1}\right), \ldots, \pi\left(w_{t}\right)\right)$ when the choice of the finite preimage $W$ of $Y$ is irrelevant.

Moreover, when the generating subset $X \subset \Gamma$ is clear from the context, we shall simply write $F_{\Gamma}$ (resp. $\left.\mathfrak{F} \varnothing l_{\Gamma}\right)$ instead of $F_{\Gamma, X}\left(\operatorname{resp} . \mathfrak{F} \varnothing l_{\Gamma, X}\right)$. 


\section{KHARLAMPOVICH GROUPS}

Theorem 1.1. Let $\Gamma=\left\langle L_{1} \cup L_{2}\right\rangle$ be a finitely generated group, $L_{1}$ and $L_{2}$ two finite disjoint subsets and respectively $H_{1}$ and $H_{2}$ the subgroups that they generate. Suppose that $H_{2}$ is amenable, $H_{1}^{\Gamma}$ is Abelian and $\Gamma=H_{1}^{\Gamma} \rtimes H_{2}$, then:

$$
A C_{n}\left(L_{1}^{A}\right) \in \mathfrak{F} \varnothing l_{\Gamma}(n), \quad \forall A \in \mathfrak{F} \varnothing l_{H_{2}}(n) .
$$

where $L_{1}^{A}=\left\{a^{-1} x a: a \in A, x \in L_{1}\right\}$.

Proof. Set $B:=C_{n}\left(L_{1}^{A}\right)$, and observe that $|A B|=|A||B|$ since $A \subset H_{2}$ and $B \subset H_{1}^{\Gamma}$ and $H_{2} \cap H_{1}^{\Gamma}=\left\{1_{\Gamma}\right\}$.

For $x \in L_{2}$ we have:

$$
\frac{|A B \backslash x A B|}{|A B|} \leq \frac{|A \backslash x A||B|}{|A||B|} \leq n^{-1}
$$

For $x \in L_{1}$, using Lemma 3, we have:

$$
\begin{aligned}
& \frac{|A B \backslash x A B|}{|A B|}=\frac{|\{a b: a \in A, b \in B: a b \notin x A B\}|}{|A||B|}= \\
& =\frac{\left|\left\{a b: a \in A, b \in B: b \notin a^{-1} x A B\right\}\right|}{|A||B|} \leq \frac{\left|\left\{a b: a \in A, b \in B: b \notin a^{-1} x a B\right\}\right|}{|A||B|} \leq \\
& \leq \frac{\left|\bigcup_{a \in A} a\left(B \backslash a^{-1} x a B\right)\right|}{|A||B|} \leq n^{-1}\left(\text { since } a^{-1} x a \in L_{1}^{A} \text { and } B=C_{n}\left(L_{1}^{A}\right)\right) .
\end{aligned}
$$

Consider the description of a Kharlampovich group $G(M)$ given in [13], with $M$ a Minsky machine with unsolvable halting problem and $p$ a fixed prime, using the same notation of [13], we have:

- $H_{j}:=\left\langle L_{j}\right\rangle, j=0,1,2$, and $H:=\left\langle L_{1} \cup L_{2}\right\rangle$,

- $H_{j}$ is abelian, $j=0,1,2$;

- $H_{0}, H_{1}$ are of exponent $p$;

- $H_{1}^{H}$ is abelian of exponent $p$;

- $H=H_{1}^{H} \rtimes H_{2}$;

- $H_{0}^{G(M)}$ is abelian of exponent $p$;

- $G(M)=H_{0}^{G(M)} \rtimes H$.

Then by Theorem 1.1 we have:

$$
\begin{gathered}
C_{n}\left(L_{2}\right) \in \mathfrak{F} \varnothing l_{H_{2}}(n), \\
C_{n}\left(L_{2}\right) C_{n}\left(L_{1}^{C_{n}\left(L_{2}\right)}\right) \in \mathfrak{F} \varnothing l_{H}(n),
\end{gathered}
$$


since $H_{1}^{H}$ is of exponent $p$, then for $n \geq p$ we have $C_{n}=C_{p}$ in $H_{1}^{H}$ and the same holds in $H_{0}^{G(M)}$, thus:

$$
C_{n}\left(L_{2}\right) C_{p}\left(L_{1}^{C_{n}\left(L_{2}\right)}\right) C_{p}\left(L_{0}^{C_{n}\left(L_{2}\right) C_{p}\left(L_{1}^{C_{n}\left(L_{2}\right)}\right)}\right) \in \mathfrak{F} \varnothing l_{G(M)}(n) .
$$

The groups $G(M)$ have computable Følner sets: we have an algorithm with input $n$ and output a finite subset of the free group projecting onto an $n$-Følner set in $G(M)$. The Theorem A of Introduction is proved. Moreover, we have a bound from above for the cardinality of the smallest Følner sets for $G(M)$.

Corollary 1.2. The class of finitely presented groups with computable Følner sets is larger than the class of finitely presented amenable groups with solvable Word Problem.

\section{Corollary 1.3.}

$$
F_{G(M)}(n) \leq n^{\left|L_{2}\right|} p^{\left|L_{1}\right| n^{\left|L_{2}\right|}} p^{\left|L_{0}\right| n^{\left|L_{2}\right|} p^{\left|L_{1}\right| n^{\left|L_{2}\right|}}}
$$

\section{Abelian EXTENSION}

We consider now the general Abelian extensions: a priori the procedure doesn't ensure computability of the Følner sets in every case.

Theorem 2.1. Let $\Gamma$ be finitely generated by $X$. Suppose $N \triangleleft \Gamma$ is an Abelian normal subgroup and denote by $\rho: \Gamma \rightarrow \Gamma / N$ the canonical projection. Then

$$
A C_{2 n|A|^{2}}\left(A^{-1} X A \cap N\right) \in \mathfrak{F} \varnothing l_{\Gamma, X}(n),
$$

for each finite $A \subset \Gamma$ such that $|A|=|\rho(A)|$ and $\rho(A) \in \mathfrak{F}_{\Gamma / N, \rho(X)}(2 n)$.

Proof. Consider the finite set $S:=A^{-1} X A \cap N$ and, for each $x \in X$, the finite set $S_{x}:=$ $A^{-1} x A \cap N$. We clearly have $|S| \leq|A|^{2}|X|$ and $\left|S_{x}\right| \leq|A|^{2}$.

Set $B:=C_{2 n|A|^{2}}(S) \subset N$. Then by Lemma 3 we have $\frac{|B \backslash s B|}{|B|} \leq\left(2 n|A|^{2}\right)^{-1}$ for all $s \in S$; thus for any $s \in S_{x}$, for any $x \in X$

$$
\frac{|B \backslash s B|}{|B|} \leq\left(2 n\left|S_{x}\right|\right)^{-1}
$$

Consider the set $F:=A B \subset \Gamma$ and notice that $|F|=|A||B|$ because the intersection $A \cap B$ has at most one element since $\rho_{\left.\right|_{A}}$ is injective and $\rho$ sends $B$ to the identity of $\Gamma / N$. So for $g \in F$ we write $g=a b, a \in A, b \in B$ in a unique way (again because $\rho_{\left.\right|_{A}}$ is injective and $\rho(g)=\rho(a)$ ) 
and we write $A^{\prime}:=\rho(A) \subset \Gamma / N$, recall that this is $2 n$-Følner in $\Gamma / N$.

For each $x \in X$, the set $F \backslash x F$ is the disjoint union of the subsets:

$$
\begin{aligned}
& E_{1}^{x}=\left\{g \in F \backslash x F: \rho(g) \notin \rho(x) A^{\prime}\right\} \\
& E_{2}^{x}=\left\{g \in F \backslash x F: \rho(g) \in \rho(x) A^{\prime}\right\} .
\end{aligned}
$$

If $g=a b \in E_{1}^{x}$, since $\rho(g)=\rho(a) \notin \rho(x) A^{\prime}$ we have $\rho(a) \in A^{\prime} \backslash \rho(x) A^{\prime}$. But $\rho$ is injective on $A$ then:

$$
\frac{\left|E_{1}^{x}\right|}{|F|}=\frac{\left|A^{\prime} \backslash \rho(x) A^{\prime}\right||B|}{|A||B|} \leq(2 n)^{-1} .
$$

If $g=a b \in E_{2}^{x}$ then $\rho(a) \in \rho(x) A^{\prime}=\rho(x A)$. Hence there exist $a^{\prime} \in A, s \in N$ such that $a s=x a^{\prime}$. It follow that $s=a^{-1} x a^{\prime}$ and $s \in S_{x}$. Now $g=x a^{\prime} s^{-1} b$, and since $g \notin x F=x A B$ we necessarily have $b \notin s B$. Thus we have

$$
\frac{\left|E_{2}^{x}\right|}{|F|} \leq \frac{\left|\left\{x a^{\prime} s^{-1} b, a^{\prime} \in A, s \in S_{x}, b \in B \backslash s B\right\}\right|}{|A||B|} \leq \sum_{s \in S_{x}} \frac{|B \backslash s B|}{|B|}
$$

And by (3):

$$
\frac{\left|E_{2}^{x}\right|}{|F|} \leq(2 n)^{-1}
$$

Combining (4) and (5) we deduce that $\frac{|F \backslash x F|}{|F|}=\frac{\left|E_{1}^{x}\right|}{|F|}+\frac{\left|E_{2}^{x}\right|}{|F|} \leq n^{-1}$, for any $x \in X$.

Corollary 2.2. A finitely presented group which is the extension of an amenable group with solvable Word Problem by an Abelian group has computable Følner sets.

Proof. Consider the case of $\Gamma / N$ amenable with solvable Word Problem and with the set $\rho(X)$ as generators. If $\pi_{\Gamma / N}: \mathbb{F}_{X} \rightarrow \Gamma / N$ is the canonical epimorphism, for every $n$ we can compute $\mathcal{A} \in \mathbb{F}_{X}$ such that $\pi_{\Gamma / N}(\mathcal{A}) \in \mathfrak{F} \varnothing l_{\Gamma / N, \rho(X)}(2 n)$, but also with $|\mathcal{A}|=\left|\pi_{\Gamma / N}(\mathcal{A})\right|$, by the solvability of the Word Problem.

But then $A:=\pi_{\Gamma, X}(\mathcal{A})$ is such that $\rho(A)=\pi_{\Gamma / N}(\mathcal{A}) \in \mathfrak{F} \varnothing l_{\Gamma / N, \rho(X)}(2 n)$ and $|A|=|\rho(A)|$, because:

$$
|\rho(A)| \leq|A| \leq|\mathcal{A}|=\left|\pi_{\Gamma / N}(\mathcal{A})\right|
$$

Moreover, given an element $\omega \in \mathcal{A}^{-1} X \mathcal{A}$ we can compute if $\pi_{\Gamma / N}(\omega)=1_{\Gamma / N}$ or not, and then we can compute the preimage of $A^{-1} X A \cap N$ in $\mathbb{F}_{X}$ and finally we can compute a preimage of the $n$-Følner sets for $\Gamma$.

This implies again that Kharlampovich groups have computable Følner sets, because they are Abelian extensions of finitely presented metabelian, and therefore residually finite with solvable WP, groups.

Notice that the Abelian group $N$ may be not finitely generated. 
Corollary 2.3. If $\Gamma$ is finitely generated by $X$ and $N \triangleleft \Gamma$ is an Abelian normal subgroup, denoting with $\rho: \Gamma \rightarrow \Gamma / N$ the projection:

$$
F_{\Gamma}(n) \leq F_{\Gamma / N}(2 n)\left(2 n F_{\Gamma / N}(2 n)^{2}\right)^{|X| F_{\Gamma / N}(2 n)^{2}}
$$

Proof. We consider $\rho(A) \in \mathfrak{F} \varnothing l_{\Gamma / N}(2 n)$ such that $|\rho(A)|=|A|=F_{\Gamma / N}(2 n)$, recall that $S=$ $A^{-1} X A \cap N$ and then $|S| \leq|X||A|^{2}$.

\section{SPLITTING EXTENSIONS}

The situation is clearer if the extension splits. In this case we can also consider extensions by amenable groups.

Theorem 3.1. Let $N$ and $H$ be groups respectively generated by the finite sets $Z$ and $Y$, let $\phi: H \rightarrow \operatorname{Aut}(N)$ be a homomorphism. Let $c:=\max \left\{\left|\phi_{y}(z)\right|_{Z}: z \in Z, y \in Y\right\}$.

Then if $A \in \mathfrak{F} \varnothing l_{H, Y}(n)$ and $B \in \mathfrak{F} \varnothing l_{N, Z}\left(n c^{|A|_{Y}}\right)$ we have

$$
A B \in \mathfrak{F} \varnothing l_{N \rtimes_{\phi} H, Z \cup Y}(n),
$$

(recall that $\left.|A|_{Y}=\max \left\{|a|_{Y}: a \in A\right\}\right)$.

Proof. We first observe that $|A B|=|A||B|$ because $A \subset H$ and $B \subset N$.

For $y \in Y(\subset H)$ we have:

$$
\frac{|A B \backslash y A B|}{|A B|} \leq \frac{|A \backslash y A||B|}{|A||B|} \leq n^{-1}
$$

For $z \in Z(\subset N)$ we have:

$z a b=a a^{-1} z a b=a \phi_{a}(z) b$, so that $\{a b \in A B: z a b \notin A B\} \subset\left\{a b \in A B: \phi_{a}(z) b \notin B\right\}$. We deduce

$$
\frac{|A B \backslash z A B|}{|A B|} \leq \frac{\left|\bigcup_{a \in A} a\left[B \backslash \phi_{a}(z) B\right]\right|}{|A||B|} \leq \frac{\sum_{a \in A}\left|B \backslash \phi_{a}(z) B\right|}{|A||B|} .
$$

Since $\left|\phi_{a}(z)\right|_{Z} \leq c^{|a|_{Y}} \leq c^{|A|_{Y}}$ then, using Lemma 1:

$$
\frac{\sum_{a \in A}\left|B \backslash \phi_{a}(z) B\right|}{|A||B|} \leq \frac{\left|\phi_{a}(z)\right|_{Z}}{c^{|A|_{Y}} n} \leq n^{-1},
$$

because $B \in \mathfrak{F} \varnothing l_{N}\left(n c^{|A|_{Y}}\right)$.

Corollary 3.2. The semidirect product of two finitely generated groups with computable Følner sets has computable Følner sets. 
Proof. We can compute $\mathcal{A}$, the preimage of a $n$-Følner set $A$ for $H$, we compute $m$, the maximal length of words in $\mathcal{A}$ in the free group. We compute $\mathcal{B}$, the preimage of $B \in \mathfrak{F} \varnothing l_{N}\left(n c^{m}\right)$. Since $|A|_{Y} \leq m$ we have $B \in \mathfrak{F} \varnothing l_{N}\left(n c^{|A|_{Y}}\right)$ and then by Theorem 3.1 we have that $\mathcal{A B}$ is a preimages of an $n$-Følner set for the semidirect product.

Corollary 3.3. In the same hypotheses of the above theorem:

$$
F_{N \rtimes_{\phi} H}(n) \leq F_{H}(n|Y|) F_{N}\left(n c^{F_{H}(n|Y|)}\right) .
$$

Proof. By Lemma 2 we have $A \in \mathfrak{F} \varnothing l_{H}(n)$ with $|A|_{Y} \leq|A| \leq F_{H}(|Y| n)$ then we choose the optimal $B \in \mathfrak{F} \varnothing l_{N}\left(n c^{F_{H}(n|Y|)}\right)$. Clearly $B \in \mathfrak{F} \varnothing l_{N}\left(n c^{|A|_{Y}}\right)$.

\section{GENERAL EXTENSIONS}

Theorem 4.1. Let $\Gamma$ be generated by the finite set $X$ and $N$ be a normal subgroup of $\Gamma$ generated by the finite set $Y$. Let $\rho: \Gamma \rightarrow K:=\Gamma / N$ be the projection to the quotient. For any finite subset $A \subset \Gamma$ such that $A^{\prime}:=\rho(A) \in \mathfrak{F} \varnothing l_{K, \rho(X)}(2 n)$, with $|A|=\left|A^{\prime}\right|$ and $|A|_{X} \leq\left|A^{\prime}\right|_{\rho(X)}$, and any $B \in \mathfrak{F} \varnothing l_{N, Y}\left(2 n\left|A^{\prime}\right|^{2} \Delta_{N}^{\Gamma}\left(2\left|A^{\prime}\right|_{\rho(X)}+1\right)\right)$ we have

$$
A B \in \mathfrak{F} \phi l_{\Gamma, X}(n) .
$$

Proof. Setting $F:=A B$ it is easy to see that $|F|=\left|A^{\prime}\right||B|$ because $\rho$ is injective on $A$.

For each $x \in X$, the set $F \backslash x F$ is the disjoint union of the sets $E_{1}^{x}$ and $E_{2}^{x}$, defined by:

$$
\begin{aligned}
& E_{1}^{x}=\left\{g \in F \backslash x F: \rho(g) \notin \rho(x) A^{\prime}\right\} \\
& E_{2}^{x}=\left\{g \in F \backslash x F: \rho(g) \in \rho(x) A^{\prime}\right\} .
\end{aligned}
$$

We can write $g=a b$, with $a \in A$ and $b \in B$, in a unique way.

If $g \in E_{1}^{x}$, since $\rho(g)=\rho(a) \notin \rho(x) A^{\prime}$ we have $\rho(a) \in A^{\prime} \backslash \rho(x) A^{\prime}$. Moreover, since $\rho$ is injective on $A$ :

$$
\frac{\left|E_{1}^{x}\right|}{|F|}=\frac{\left|A^{\prime} \backslash \rho(x) A^{\prime}\right||B|}{\left|A^{\prime}\right||B|} \leq(2 n)^{-1} .
$$

If $g \in E_{2}^{x}$ then $\rho(g)=\rho(a) \in \rho(x) A^{\prime}$ so that there exists $a^{\prime} \in A$ satisfying $\rho(a)=\rho(x) \rho\left(a^{\prime}\right)$. The images by $\rho$ of $a$ and $x a^{\prime}$ are the same so we can find $s \in N$ such that $a s=x a^{\prime}$.

Setting $S_{x}:=A^{-1} x A \cap N$ we see that $s \in S_{x}$ and $\left|S_{x}\right| \leq|A|^{2}$. Then $g=x a^{\prime} s^{-1} b$, and since $g \notin x A B$ we deduce that $b \notin s B$. It follows that:

$$
\frac{\left|E_{2}^{x}\right|}{|F|} \leq \frac{\left|\left\{x a^{\prime} s^{-1} b, a^{\prime} \in A, s \in S_{x}, b \in B \backslash s B\right\}\right|}{\left|A^{\prime}\right||B|} \leq \sum_{s \in S_{x}} \frac{|B \backslash s B|}{|B|} .
$$

We have a bound for $\left|S_{x}\right|$; we need a bound for the length of the elements in $S_{x}$. For every $s \in S_{x}$ we have: 
$|s|_{Y} \leq \Delta_{N}^{\Gamma}\left(|s|_{X}\right)$. On the other hand, $|s|_{X}=\left|a^{-1} x a^{\prime}\right|_{X} \leq 2|A|_{X}+1 \leq 2\left|A^{\prime}\right|_{\rho(X)}+1$.

From Lemma 1 we then deduce:

$$
\frac{|B \backslash s B|}{|B|} \leq\left(2 n\left|A^{\prime}\right|^{2}\right)^{-1} \leq \frac{1}{2 n\left|S_{x}\right|}
$$

Finally $\frac{|F \backslash x F|}{|F|}=\frac{\left|E_{1}^{x}\right|}{|F|}+\frac{\left|E_{2}^{x}\right|}{|F|} \leq n^{-1}$, showing that $F$ is the an $n$-Følner set.

Corollary 4.2. Let $N, \Gamma, K$ finitely generated groups such that:

$$
1 \rightarrow N \rightarrow \Gamma \rightarrow K \rightarrow 1
$$

If $N$ has computable Følner sets, $\Delta_{N}^{\Gamma}$ is subrecursive, $K$ is amenable with solvable Word Problem, then $\Gamma$ has computable Følner sets.

Proof. $N$ and $K$ have computable Følner sets. For each $k$ we can construct $\mathcal{A} \subset \mathbb{F}_{X}$ such that $\pi_{K}(\mathcal{A}) \in \mathfrak{F} \varnothing l_{K}(k)$. We denote $A^{\prime}:=\pi_{K}(\mathcal{A})$. If we consider $A:=\pi_{\Gamma, X}(\mathcal{A})$, it is clear that $\rho(A)=A^{\prime} \in \mathfrak{F} \varnothing l_{K}(k)$. If $K$ has solvable Word Problem we can detect $\mathcal{A}$ such that $\pi_{K}$ is injective on $\mathcal{A}$ and $|\omega|=\left|\pi_{K}(\omega)\right|_{\rho(X)}$ for every $\omega \in \mathcal{A}$. So we can compute a preimage for a set $A$ respecting the hypotheses of the Theorem 4.1. For the set $B$ we just need the computability (of a bound) of the number $2 n\left|A^{\prime}\right|^{2} \Delta_{N}^{G}\left(2\left|A^{\prime}\right|_{\rho(X)}+1\right)$, so if $\Delta_{N}^{\Gamma}$ is subrecursive we have the thesis.

Finally, from Theorem 4.1 and again using Lemma 2:

Corollary 4.3. Let $N, \Gamma, K$ finitely generated groups such that:

$$
1 \rightarrow N \rightarrow \Gamma \rightarrow K \rightarrow 1
$$

Then

$$
F_{\Gamma}(n) \leq F_{K}(|X| n) F_{N}\left(2 n F_{K}(|X| n)^{2} \Delta_{N}^{\Gamma}\left(2 F_{K}(|X| n)+1\right)\right) .
$$

Thus if $N$ and $K$ have subrecursive Følner function and if $\Delta_{N}^{\Gamma}$ is subrecursive then $\Gamma$ has subrecursive Folner function as well.

\section{REFERENCES}

[1] G. N. Arzhantseva and D. V. Osin, Solvable groups with polynomial Dehn functions, Trans. Amer. Math. Soc. 354 (2002), no. 8, 3329-3348, DOI 10.1090/S0002-9947-02-02985-9. MR1897402 (2003b:20059)

[2] Matteo Cavaleri, Algorithms and quantifications in amenable and sofic groups, 2016. Thesis (Ph.D.)-Sapienza Università di Roma.

[3] _ Folner functions and the generic Word Problem for finitely generated amenable groups, available at arXiv:1703.04133 [math.GR].

[4] Tullio Ceccherini-Silberstein and Michel Coornaert, Cellular automata and groups, Springer Monographs in Mathematics, Springer-Verlag, Berlin, 2010. MR2683112 (2011j:37002) 
[5] Michel Coornaert, Topological dimension and dynamical systems, Universitext, Springer-Verlag, Berlin, 2015 (English).

[6] Tara C. Davis and Alexander Yu. Olshanskii, Relative subgroup growth and subgroup distortion, Groups Geom. Dyn. 9 (2015), no. 1, 237-273, DOI 10.4171/GGD/312. MR3343353

[7] Anna Erschler, Piecewise automatic groups, Duke Math. J. 134 (2006), no. 3, 591-613, DOI 10.1215/S00127094-06-13435-X. MR2254627 (2007k:20086)

[8] _ On isoperimetric profiles of finitely generated groups, Geom. Dedicata 100 (2003), 157-171, DOI 10.1023/A:1025849602376. MR2011120 (2004j:20087)

[9] Rostislav Grigorchuk, Solved and unsolved problems around one group, Infinite groups: geometric, combinatorial and dynamical aspects, Progr. Math., vol. 248, Birkhäuser, Basel, 2005, pp. 117-218, DOI 10.1007/3-76437447-0-5. MR2195454 (2007d:20001)

[10] Misha Gromov, Entropy and isoperimetry for linear and non-linear group actions, Groups Geom. Dyn. 2 (2008), no. 4, 499-593, DOI 10.4171/GGD/48. MR2442946 (2010h:37011)

[11] O. G. Harlampovič, A finitely presented solvable group with unsolvable Word Problem, Izv. Akad. Nauk SSSR Ser. Mat. 45 (1981), no. 4, 852-873, 928 (Russian).

[12] David Janzen, Følner nets for semidirect products of amenable groups, Canad. Math. Bull. 51 (2008), no. 1, 60-66, DOI 10.4153/CMB-2008-008-7. MR2384739 (2009e:43001)

[13] O. Kharlampovich, A. Myasnikov, and M. Sapir, Algorithmically complex residually finite groups, available at arXiv:1204.6506 [math.GR].

[14] Roger C. Lyndon and Paul E. Schupp, Combinatorial group theory, Classics in Mathematics, Springer-Verlag, Berlin, 2001. Reprint of the 1977 edition. MR1812024 (2001i:20064)

[15] Anatoly Ivanovich Malcev, Algorithms and recursive functions, Nauka, Moscow, 1965.

[16] Justin Tatch Moore, Fast growth in the Følner function for Thompson's group F, Groups Geom. Dyn. 7 (2013), no. 3, 633-651, DOI 10.4171/GGD/201. MR3095713

[17] Alexander Yu. Olshanskii and Denis V. Osin, A quasi-isometric embedding theorem for groups, Duke Math. J. 162 (2013), no. 9, 1621-1648, DOI 10.1215/00127094-2266251. MR3079257

[18] Laurent Saloff-Coste and Tianyi Zheng, Random walks on free solvable groups, Math. Z. 279 (2015), no. 3-4, 811-848, DOI 10.1007/s00209-014-1395-2. MR3318252

[19] A. Vershik, Amenability and approximation of infinite groups, Selecta Math. Soviet. 2 (1982), no. 4, 311-330. Selected translations. MR721030

(M. Cavaleri) Institute of Mathematics of the Romanian Academy, 21 Calea Grivitei Street, 010702 BUCHAREST, ROMANIA

E-mail address: matte.cavaleri@gmail.com 